\title{
SYMPTOMATIC AND ASYMPTOMATIC SMOKERS WITHOUT AIRFLOW OBSTRUCTION: A NeW CLINICAL ENTITY?
}

\author{
Rafael de Jesús Hernández-Zenteno ${ }^{1 *}$, Fernando Flores-Trujillo ${ }^{1}$, Jesús Javier Vázquez-Cortés², \\ Carlos Espinosa de los Monteros-Sánchez ${ }^{1}$ and Manuel G. Cosío ${ }^{3}$ \\ ${ }^{1}$ Instituto Nacional de Enfermedades Respiratorias Ismael Cosío Villegas, Mexico City, Mexico; ${ }^{2}$ Hospital Ángeles \\ Lomas, Estado de Mexico, Mexico; ${ }^{3}$ Department of Cardiac, Thoracic, and Vascular Sciences, University of Padova, \\ Padova, Italy
}

\begin{abstract}
Non-obstructed ever-smokers, with or without symptoms, have generated a great deal of information recently, but few reviews. Even individuals with normal spirometry can present changes in sputum with inflammatory biomarkers (cellular and molecular) and airways and parenchyma with remodeling; when symptomatic (cough, sputum, wheezing, and dyspnea) exacerbations are frequent affecting the individuals' quality of life, there is an increased use of health resources: more medication, emergency visits, and hospital admissions. Non-obstructed smokers may have exercise limitations, increased lung volumes, low diffusion capacity, air entrapment, peripheral airways obstruction, elevated airways resistance, and abnormal multiple breath nitrogen washout, as well as abnormalities in computed tomography studies, such as airway wall thickening, emphysema, or interstitial lung abnormalities. Quitting smoking comprises a first, inexpensive, and often abandoned intervention to arrest respiratory impairment. It is controversial whether or not this population should be treated with other medications. Further studies should be conducted to elucidate the consequences of follow-up and prognosis in this clinical entity. (REV INVEST CLIN. 2019;71:64-9)
\end{abstract}

Key words: Normal smokers. Healthy smokers. Non-obstructed smokers. Symptomatic smokers. Global initiative for chronic obstructive lung disease 0.

Corresponding author:

*Rafael de Jesús Hernández-Zenteno

Instituto Nacional de Enfermedades Respiratorias

Ismael Cosío Villegas (INER)

Calzada de Tlalpan, 4502

Col. Sección XVI, Del. Tlalpan

C.P. 14080, Mexico City, Mexico

E-mail: rafherzen@yahoo.com.mx 


\section{INTRODUCTION}

Individuals who smoke cigarettes or who smoked in the past may develop a variety of respiratory alterations and diseases. Chronic-obstructive pulmonary disease (COPD) is one of the consequences of smoking, causing millions of deaths and disease in the world, and is the third most common cause of death worldwide. However, smokers may develop diseases not conforming to the classical COPD definition with airflow obstruction detected by spirometry. For example, smokers may develop chronic bronchitis, chronic cough or phlegm, or be without airflow obstruction, that is, non-obstructive chronic bronchitis. Individuals with emphysema detected on computed tomography (CT) may also lack airflow obstruction. Recently, this population of smokers without airflow obstruction has been characterized again and is the theme of this review.

Airflow obstruction is defined by spirometry as a reduced post-bronchodilator forced expiratory volume at one second $\left(\mathrm{FEV}_{1}\right) /$ forced vital capacity (FVC) ratio, and low $\mathrm{FEV}_{1} / \mathrm{FVC}$ was defined by the global initiative for chronic obstructive lung disease (GOLD) criteria as $<0.70$. More recently, the GOLD classification has included symptoms and exacerbations due to their importance, and also due to the lack of accessibility to spirometry testing in different regions and countries.

The prognostic and therapeutic implications in symptomatic smokers without COPD, beyond the fact that they should urgently suspend smoking as a first priority, are a controversial field that remains under discussion. This is because symptoms do not precisely and necessarily predict the development of COPD: $80 \%$ of smokers evade COPD and another percentage of subjects lose pulmonary function despite being asymptomatic. One perspective is focused on how smokers avoid COPD in terms of the mechanisms of inflammation and the immune response, and the second is centered on the observation of several cohorts, defining the proportion of the different populations that acquired COPD over a long time-period. It is not clear, which is the percentage of smokers who have a number of symptoms (cough, sputum, wheezing, and dyspnea), as well as abnormalities in other tests such as imaging, lung function, and inflammation markers ${ }^{1,2}$.
There is less information available on changes in inflammatory variables such as biomarkers and the histology/morphology in airways and parenchyma of non-obstructed smokers compared with normal nonsmokers and COPD patients. Likewise, scarce information has been published on lung function testing, apart from spirometry, carbon monoxide diffusion capacity (DLCO), small airways' obstruction by impulse oscillometry, pulmonary volumes, and exercise performance in this type of smokers. Furthermore, information is scant regarding the frequency of emphysema, airways' remodeling, air trapping, bronchiectasis, and fibrosis in this group ${ }^{1,2}$.

All available evidence leads not only to search for interventions to preserve lung function and quality of life and avoid exacerbations, above all, through smoking cessation, but also to find possible medications that may help to reach these objectives.

\section{INFLAMMATION AND MORPHOLOGY BEFORE THE APPEARANCE OF OBSTRUCTION}

Smokers have small airways disease (SAD), goblet cell metaplasia, increase in the proportion of bronchial gland mass, smooth muscle hypertrophy, inflammation in the walls and glands of bronchioles (macrophages and neutrophils), respiratory bronchiolitis, alveolar wall thickening, and an excess of airways $<400$ $\mu$ in diameter, correlating with the extent of centrilobular emphysema, compared with non-smokers ${ }^{3-5}$. For most pathologic features examined (inflammation, fibrosis, muscle scores, and goblet cells number), there is an orderly progression in severity when comparing smokers to non-smokers and COPD to smok$\operatorname{ers}^{3-5}$ (Fig. 1).

Normal (non-obstructed and asymptomatic) cigarette smokers may have an inflammatory reaction involving the entire tracheobronchial tree (central and peripheral airways, lumen, parenchyma, and pulmonary arteries) and alveolar wall. T-lymphocytes and macrophages are the predominant cells infiltrating the airway wall, whereas neutrophils, which are scarce in the airway wall, are increased in the airway lumen, while mononuclear cells, CD4 ${ }^{+}$T-Lymphocytes and clusters of macrophages are observed in the wall of peripheral airways ${ }^{6,7}$. 
Figure 1. Surgical specimen of a 78-year-old female smoker (60 packs/year) with normal spirometry (no obstruction in forced expiratory volume at one second $\left[\mathrm{FEV}_{1}\right]=115 \% \mathrm{p}$ and $\mathrm{FEV}_{1} /$ forced vital capacity ratio $=0.85$ ), normal diffusion capacity $(88 \% \mathrm{p})$, and air entrapment in plethysmography (residual volume $=178 \% \mathrm{p}$ ), whose sample shows small airway disease (airway wall thickness, epithelial metaplasia, basement membrane thickness, smooth muscle hypertrophy, and outer lawyer fibrosis) and parenchymal emphysema (destroyed alveolar attachments and enlarged airspace).

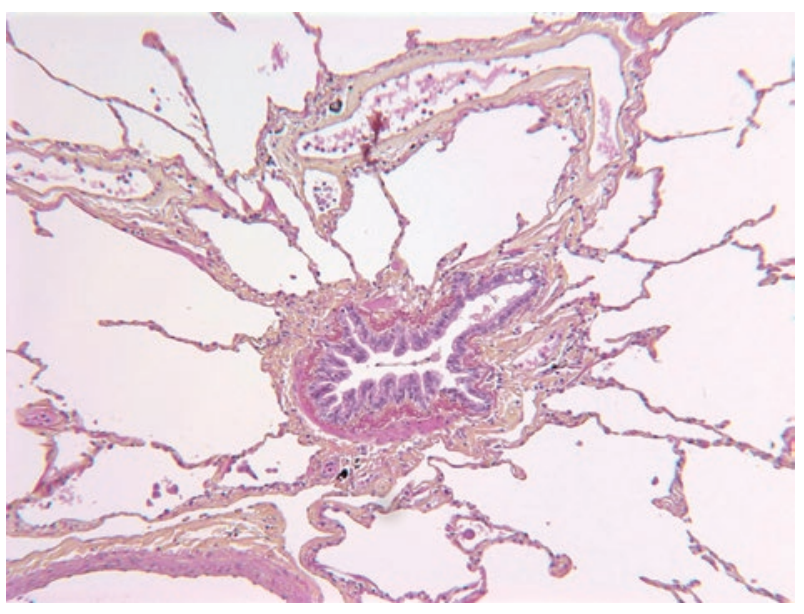

In healthy, non-obstructed smokers, the number of $\mathrm{CD}^{+}{ }^{+}$T-lymphocytes showed a significant negative correlation with $\mathrm{FEV}_{1}$ as did the value of smooth muscle area normalized by the internal perimeter, in the same way, that occurs in COPD smokers ${ }^{8}$. For a given perimeter of the basement membrane, the luminal diameter, total wall area, smooth muscle area, destroyed alveolar attachments and airway external perimeters are similar in non-obstructed smokers and COPD smokers. Airways' responsiveness and air trapping are associated with airway luminal size, total airway wall thickness and airway smooth muscle thickness in non-obstructed smokers as in those with COPD $^{9}$. Sputum biomarkers relevant for airway inflammation (myeloperoxidase, neutrophil gelatinaseassociated lipocalin, YKL-40, interleukin [IL]-6), in healthy smokers have intermediate levels between those from non-smokers and those with COPD ${ }^{10}$.

\section{RESPIRATORY SYMPTOMS AND QUALITY OF LIFE IN NON-OBSTRUCTED EVER-SMOKERS}

Several studies have explored the prevalence of various respiratory symptoms and the risk factors for their appearance. In the IBERPOC study in Spain, the percentage of cigarette smokers with a cough was $31.8 \%$ and with sputum $22.7 \%$, versus $5 \%$ and $3 \%$ in non-smokers, respectively ${ }^{11}$. In another study, the risk of dyspnea (odds ratio [OR]) increased with the number of cigarettes smoked per day: 1-14 cig/day = $1.89,15-24 \mathrm{cig} /$ day $=2.98$, and $>24 \mathrm{cig} /$ day $=3.57$; women were more prone to have dyspnea Grade 3 compared to men (11.7 vs. $10.3 \%)^{12}$. Former smokers also developed dyspnea, as Grade 3 was present in up to $46.7 \%$ of men and $12.8 \%$ of women ${ }^{12}$. In a study from Iran, habitual smoking was a predictor of the presence of cough (OR, 2.27), breathlessness (1.88), phlegm (2.9), and chest tightness (1.41) compared to never smokers; this corresponds to a prevalence of cough of $41.8 \%$, phlegm $40.5 \%$, breathlessness $18.9 \%$, and chest tightness $10.6 \%{ }^{13}$. Former smokers may also be symptomatic: cough was present in $30.2 \%(O R=1.31)$, phlegm in $24.5 \%(O R=$ $1.45)$, breathlessness in $18.5 \%(O R=1.69)$, and chest tightness in $11.9 \%(\mathrm{OR}=1.56)^{13}$.

In a group of non-obstructed ever-smokers ( $42.1 \%$ still smoking), the proportion of individuals with cough and phlegm was $27.4 \%$, morning cough $20 \%$, chronic cough $15.6 \%$, wheezing attacks $8.3 \%$, and dyspnea Grade $>1$ (modified medical research council scale) was $14.5 \%^{14}$.

The quality of life in GOLD stage 0 (smokers with symptoms and normal spirometry) measured by the St George Respiratory Questionnaire (SGRQ) and the short form-36 generic scale, is significantly affected: SGRQ symptoms domain score for GOLD 0 was 27 and for non-smokers, 12.4 (the higher the score and the worse quality of life). A dyspnea score of $>1$ was present in $23.5 \%$ of GOLD 0 smokers in contrast to $3.7 \%$ of never smokers; chronic bronchitis was present in $12.6 \%$ of GOLD 0 and $4.3 \%$ had severe exacerbations compared to none of never smokers ${ }^{15}$.

In a study of 2736 current or former smokers and never-smoker controls to whom the COPD assessment test (CAT) was applied, respiratory symptoms were present in $50 \%$ of current or former smokers with preserved pulmonary function, an intermediate prevalence between never smokers and GOLD mild/ moderate group ${ }^{16}$. The prevalence of chronic bronchitis in asymptomatic current or former smokers with CAT $<10$ was $6 \%$ and in those with CAT $\geq 10$ was 
$33 \%$; likewise, for wheezing was $32 \%$ and $69 \%$ in current or former smokers with CAT $<10$ or $\geq 10$, respectively. Symptomatic current or former smokers had more respiratory exacerbations $(0.27 \pm 0.67$ mean and standard deviation events per year) than asymptomatic current or former smokers (0.08 \pm $0.31)$ and never smokers $(0.03 \pm 0.21)(p<0.001$ for both comparisons) ${ }^{16,17}$.

Respiratory symptoms are often treated although no medication recommendation exists: $20 \%$ of symptomatic GOLD 0 or impaired smokers used a respiratory medication (inhaled long and short $\beta$ agonist $17.3 \%$, inhaled anticholinergics $6.6 \%$, and inhaled corticosteroids $7.7 \%$ ); and $5 \%$ of asymptomatic GOLD 0 used a respiratory medication $(4.2 \%, 0.6 \%$, and $1.8 \%$ according to the kind of inhaled medication, respectively). GOLD 0 individuals who were users of respiratory medications were more commonly African-American women, with chronic bronchitis, more exacerbations, and dyspnea ${ }^{15}$.

Among symptomatic (CAT $\geq 10$ ) current or former smokers, $42 \%$ used bronchodilators and $23 \%$ used inhaled glucocorticoids; other medications were used less commonly and lacking a solid basis: antibiotics and antibiotic/glucocorticoid combination. These patients have more visits to medical offices and hospitals than asymptomatic (CAT $<10$ ) current or former smokers, and current or former smokers with COPD with CAT $<10^{16}$.

\section{PULMONARY FUNCTION AND PHYSICAL ACTIVITY IMPLICATIONS IN NON-OBSTRUCTED SMOKERS}

It has been clear for a long time that ever-smokers may often have respiratory symptoms and normal spirometry, but it should be investigated whether they have other changes in lung function, since spirometry evaluates only mechanical alterations. Pulmonary compliance (static pressure-volume) and total lung capacity measured in a body plethysmograph increase rapidly in cigarette smokers ${ }^{18}$.

In a group of current and former smokers, those with CAT $<10$ walked $461 \mathrm{~m}$ in the 6 -minute walk test (6MWT) compared with $410 \mathrm{~m}$ among those with CAT > 10. Non-obstructed smokers (or ex-smokers) had shorter 6MWT (447 m) than never smokers (493 $\mathrm{m})^{15}$. On the other hand, in another study, DLCO was normal $(7.5 \mathrm{mmol} / \mathrm{min} / \mathrm{KPa})$ in non-obstructed smokers even though $42 \%$ were still smoking ${ }^{14}$. In a cohort study of non-obstructed ever-smokers (25\% active smokers) with normal or low diffusion capacity (DLCO $=68 \% \mathrm{p}$ ), after 3 years of follow-up, $22 \%$ of those with low DLCO developed obstruction, compared with $3 \%$ of the normal spirometry/normal DLCO group ${ }^{19}$.

Symptomatic ever-smokers, regardless of asthma history, had slightly lower $\mathrm{FEV}_{1}(94.1 \% \mathrm{p})$ and response to bronchodilator (7.4\%) than asymptomatic eversmokers (98.5\%p and 6.1, respectively), with greater limitation of activity ${ }^{16}$.

Respiratory symptoms and peripheral airway dysfunction are common in smokers with normal spirometry: $76 \%$ of the subjects had an abnormality either in the multiple breath nitrogen washout or the impulse oscillometry system $(\mathrm{IOS})^{20}$. Chronic bronchitis symptoms were associated with conductive airway abnormalities, while wheeze was associated with an increased resistance. In another study of non-obstructed smokers, the prevalence of SAD evaluated by IOS was $26.3 \%$, and they had lower spirometry values, poorer quality of life, and higher levels of C-reactive protein $^{21}$. Abnormal IOS test has also been associated with inflammation in bronchoalveolar lavage: two-fold higher lymphocyte and neutrophil counts and higher IL-8, eotaxin, and fractalkine levels. Reactivity of distal obstruction and reactance by IOS correlated with levels of IL-8, eotaxin, fractalkine, IL-12p70, and transforming growth factor- $\alpha^{22}$.

\section{IMAGE CHARACTERISTICS AND FINDINGS IN SMOKERS}

$\mathrm{CT}$ of the thorax is more sensitive for detecting lung abnormalities, and current software allows to perform accurate airway and parenchymal measurements, such as airway wall thickness, and calculate the percentage of lung with emphysema (low attenuation areas, density < -95 HU, \% LAA-950). GOLD 0 smokers have been shown to have thicker airways than never smokers (32.2 vs. $7.4 \%$ ). Non-obstructed smokers (GOLD 0) had more emphysema (24\%), airways thickening $(30.7 \%)$, or any of those $(42.3 \%)$ than never smokers ( $3 \%, 9 \%$, and $10 \%$, respectively) ${ }^{15}$. 
Figure 2. High-resolution computed tomography of the thorax of a 57-year-old male smoker (22 packs/year) with normal spirometry (no obstruction forced expiratory volume at one second $\left[\mathrm{FEV}_{1}\right]=80 \% \mathrm{p}$, and $\mathrm{FEV}_{1} /$ forced vital capacity ratio $=0.86)$, normal diffusion capacity $(78 \% \mathrm{p})$, air entrapment in the plethysmography (residual volume $=149 \% p$ ), and no dyspnea or desaturation in 6-minute walking test; image demonstrates centrilobular emphysema, subpleural bullae, and subsegmental airways wall thickness.

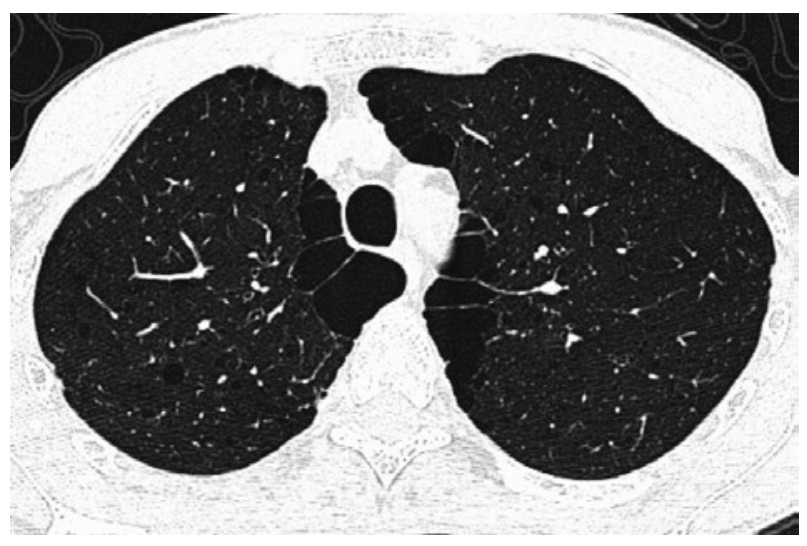

Symptomatic (CAT > 10) non-obstructed ever-smokers had more airways disease by CT not only than never smokers but also than asymptomatic eversmokers or asymptomatic mild-moderate COPD ${ }^{16}$. In this study, no increase in emphysema was seen $(<2 \%$ emphysema) in ever-smokers with preserved spirometry regardless of the CAT score ${ }^{16}$. Emphysema and increased airways wall thickness (AWT) by high-resolution CT (HRCT) were already found as remodeling manifestations in non-obstructed smokers (Fig. 2). The median $\left(25-75^{\text {th }}\right.$ percentile) percent of low-attenuation area was minimum, an extent of $0.71 \%$ and $0.32 \%$ in males and females, respectively. Interestingly, the extent of emphysema was greater in exsmokers than in current smokers. For airways findings, the mean AWT was 0.488 and $0.463 \mathrm{~mm}$ in males and females, respectively, similar to the COPD group ${ }^{23}$.

In non-obstructed smokers (current smoking of $42.12 \%$ ), the median $\left(25^{\text {th }}-75^{\text {th }}\right.$ percentile) percent low-attenuation areas < -950 HU (\% LAA-950) were also related to minimum emphysema of 0.5 , and mean AWT at an internal perimeter of $10 \mathrm{~mm}$ (AWT-Pi10) was increased in subjects without COPD. This AWT-Pi10 was significantly related to wheezing in subjects without COPD. OR for increased dyspnea was $1.9 / 10 \%$ increase in \% LAA-950 and 1.11/0.1-mm increase in AWT-Pi10 ${ }^{14}$. This remodeling, although small, was associated to symptoms.

In a large group of smokers, where $39 \%$ of participants were GOLD $=0$, interstitial lung abnormalities (ILA) were present in $8 \%$ of HRCT scans evaluated in a group with more exposure to tobacco and current smoking, more likely to have a restrictive lung deficit (total lung capacity $<80 \%$ of the predicted value; OR $=2.3$ ), and less likely to meet the diagnostic criteria for COPD (OR $=0.53)^{24}$. Those participants with GOLD $=0$ had a high prevalence of centrilobular emphysema (44\%), mixed centrilobular and subpleural emphysema (36\%), and ILA $(39 \%)^{24}$.

\section{CONCLUSIONS}

Non-obstructed smokers may have a variety of alterations in lung morphology and function and persistent inflammatory abnormalities. Respiratory symptoms and acute exacerbations in non-obstructed current or former smokers are common, with an adverse impact on quality of life. Lung function, exercise tolerance and CT scanning abnormalities such as bronchial wall thickening, or emphysema may also be present.

It is controversial whether or not this population should be treated with medications besides intensive efforts to stop smoking. Further studies should be done to elucidate follow-up and prognosis issues.

\section{REFERENCES}

1. Cosio MG, Saetta M. Evasion of COPD in smokers: at what price? Eur Respir J. 2012:39:1298-303.

2. Rodríguez-Roisin R, Han ML, Vestbo J, et al. Chronic respiratory symptoms with normal spirometry: a reliable clinical entity? Am J Respir Crit Care Med. 2017:195:17-22.

3. Cosio MG, Hale KA, Niewoehner DE. Morphologic and morphometric effects of prolonged cigarette smoking on the small airways. Am Rev Respir Dis. 1980;122:265-21.

4. Hale KA, Ewing SL, Gosnell BA, Niewoehner DE. Lung disease in long-term cigarette smokers with and without chronic air-flow obstruction. Am Rev Respir Dis. 1984;130:716-21.

5. Maestrelli P, Saetta M, Mapp CE, Fabbri LM. Remodelling in response to infection and injury. Airway inflammation and hypersecretion of mucus in smoking subjects with chronic obstructive pulmonary disease. Am J Respir Crit Care Med. 2001; 164:S76-80

6. Saetta M, Turato G, Maestrelli P, Mapp CE, Fabbri LM. Cellular and structural bases of chronic obstructive pulmonary disease. Am J Respir Crit Care Med. 2001;163:1304-9.

7. Ballarin A, Bazzan E, Hernández-Zenteno R, et al. Mast cell infiltration discriminates between histopathological phenotypes of chronic obstructive pulmonary disease. Am J Respir Crit Care Med. 2012;186:233-9. 
8. Saetta M, Di Stefano A, Turato G, et al. CD8+ T-lymphocytes in peripheral airways of smokers with chronic obstructive pulmonary disease. Am J Respir Crit Care Med. 1998;157:822-6.

9. Corsico A, Milanese M, Baraldo S, et al. Airway hyperresponsiveness: from molecules to bedside selected contribution: small airway morphology and lung function in the transition from normality to chronic airway obstruction. J Appl Physiol. 2003; 95:441-7.

10. Gao J, Iwamoto $H$, Koskela J, et al. Characterization of sputum biomarkers for asthma-COPD overlap syndrome. Int J COPD. 2016;11:2457-65

11. Jiménez-Ruiz CA, Sobradillo V, Gabriel R, et al. Respiratory symptoms and diagnosis of COPD in smokers of various types to tobacco. Results from the IBERPOC study. Bronconeumologia. 2002;38:530-5.

12. Rosi E, Scano G. Cigarette smoking and dyspnea perception. Tob Induc Dis. 2004;2:35-42.

13. Yunesian M, Homayoun-Vash J, Asghari F, Foruzanfar $\mathrm{MH}$, Hosein-Poor AR, Farhud D. Smoking-related respiratory symptoms in Tehran: a cross-sectional study. Arch Iran Med. 2008; 11:507-14.

14. Pillai SG, Grydeland TB, Dirksen A, et al. Quantitative computed tomography measures of emphysema and airway wall thickness are related to respiratory symptoms. Am J Respir Crit Care Med. 2010;181:353-9.

15. Regan EA, Lynch DA, Curran-Everett D, et al. A clinical and radiologic disease in smoker with normal spirometry. JAMA Intern Med. 2015;175:1539-49.
16. Woodruff $\mathrm{P}$, Barr RG, Bleecker $\mathrm{E}$, et al Clinical significance of symptoms in smokers with preserved pulmonary function. $\mathrm{N}$ Eng J Med 2016;374:1811-21.

17. Dransfield M, Kunisaki KM, Strand MJ, et al. Acute exacerbations and lung function loss in smokers with and without chronic obstructive pulmonary disease. Am J Resp Crit Care Med. 2017; 195:324-30

18. Colebatch $\mathrm{HJ}, \mathrm{Ng} \mathrm{CK}$. Rate of increase in pulmonary distensibility in a longitudinal study of smokers. Thorax 1988;43:175-82.

19. Ben-Gary H, Sanders A, Strulovici-Barel Y, et al. Risk of COPD with obstruction in active smoker with normal spirometry and reduced diffusion capacity. Eur Respir J. 2015;46:1589-97.

20. Jetmalani K, Thamrin C, Farah CS, et al. Peripheral airway dysfunction and relationship with symptoms in smokers with preserved spirometry. Respirology. 2018;23:512-8.

21. Llontop C, Garcia-Quero C, Castro A, et al. Small airway dysfunction in smokers with stable ischemic heart disease. PLoS One. 2017;12:e0182858.

22. Berger KI, Pradhan DR, Goldring RM, Oppenheimer BW, Rom WN, Sega LN. Distal airway dysfunction identifies pulmonary inflammation in asymptomatic smokers. ERJ Open Res. 2016; 2:66-2016.

23. Grydeland TB, Dirksen A, Coxson HO, et al. Quantitative computed tomography: emphysema and airway wall thickness by sex, age and smoking. Eur Respir J. 2009;34:858-65.

24. Washko GR, Hunninghake GM, Fernandez IE, et al. Lung volumes and emphysema in smokers with interstitial lung abnormalities. N Engl J Med. 2011;364:897-906. 